\title{
ELECTROCHEMICAL ANALYSIS ON OUTPUT CURRENTS OF NEUTRINO ANTINEUTRINO-SENSITIVE APPARATUS
}

\author{
Nur Aida ${ }^{1 *}$ Kenji Ishibashi $^{2}$, Shouhei Nakamura ${ }^{3}$, Soya Tsuda ${ }^{4}$ and Ima \\ Hayashi $^{5}$ \\ *E-mail: nur.aida@uinjkt.ac.id
}

\section{ABSTRACT}

We analyze the interaction of antineutrinos with water particle in electrochemical detectors. We postulate that some biological products generate a scalar auxiliary field $B^{0}$ which breaks low-energy antineutrinos into boson $v_{b}$ and fermion $v_{f}$ particles. Low-energy anti neutrinos are suggested to interact with water molecules and produce output currents. We examine the output current of neutrino interactions in the electrochemical apparatus with chemical-reaction equations and half-cell model under postulated influence of weak interaction. The environmental neutrino is analysed. The output currents are treated to be generated by hydrogen ion and oxygen with the half-cell model with inclusion of weak interaction effect on hydrooxide ion recombination.

Keywords: electrochemical detector, neutrino, scalar auxiliary field

\section{INTRODUCTION}

The neutrino experiments have been performed by using electro chemical detectors under weak intraction consider. we calculated the output current of experiment by using half cell model. A neutrino is a sub atomic particle belongs to the family of leptons, which is carry no charge, has a small mass and very low probability of reaction. The theory behind the neutrino reaction, known as weak interaction. In 1934, Fermi published a theory of Beta decay. The equations describing the nuclear beta decay is written as 
Hamiltonian for weak interaction, Both of Fermi and Gamow-Teller transitions are valid in neutrino kinetics behaviour. Experimentally results of neutrino experiments proposed to consider that neutrinos are composed of constituent particles with the assumptions: Weak dipole moment and weak charge generate potential interaction, motions of neutrinos are governed by Dirac-like equation with (V)- axial-vector(AV) matrices, and neutrino mass exists as eigen value in neutrino system. Some biological materials may create the AV- type auxiliary field $B^{0}$. When the AV-type field of biological material influences to low-energy antineutrinos, it may induces them to be dissociatively ionized. This paper only describes electrochemical reactions by their assistance with weak interaction. It illustrates the basic principles at electrochemical detector of neutrino experiment.

\section{EXPERIMENTS}

In this paper we investigate an experiment by our group. The experiment was placed in the laboratory of Kyushu university Hakozaki campus under natural circumstances, The experiment was conducted for 30 days. the experiment was performed with three detectors, and results are drawn in Fig.1. 


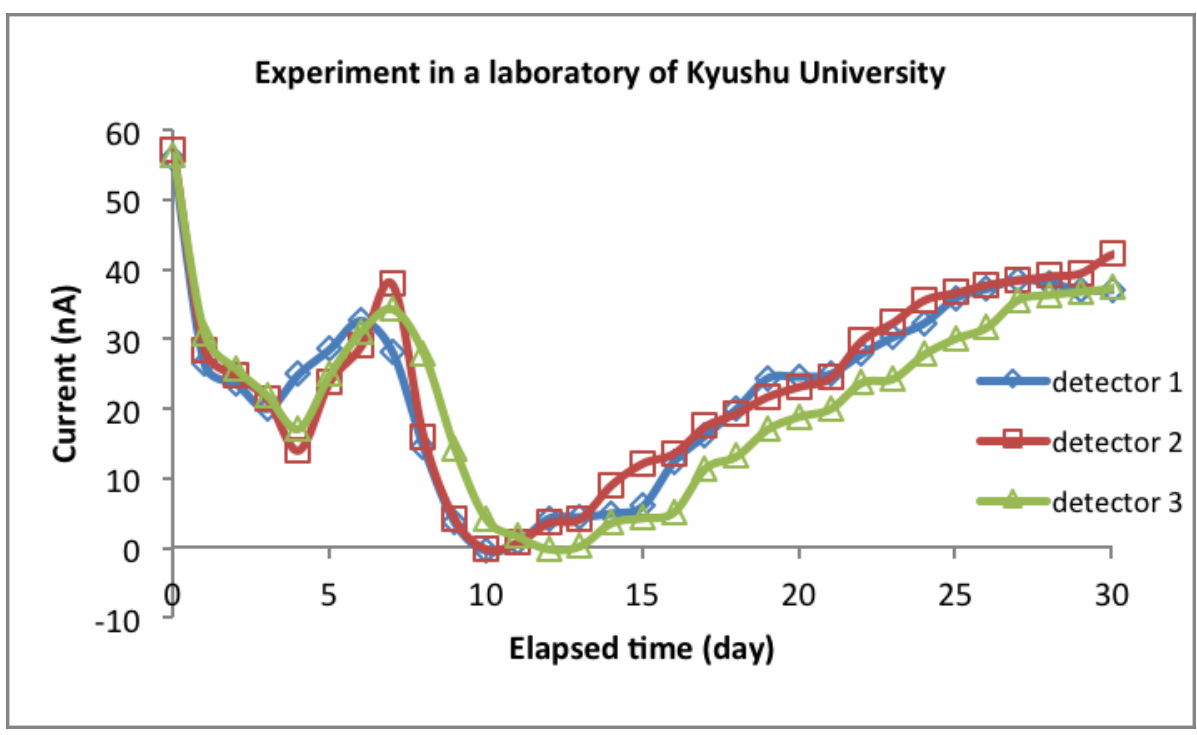

Fig. 1 Output current of experiment in a laboratory of Kyushu University under natural irradiation.

The output currents are counted from input voltage by being divided by $1 \mathrm{M}$ $\Omega$ of impedance. All detectors of the experiment show a linear trend, which provide currents decline to 15-20 nA. The initial peak values were reached at 1.5 days. Currents decrease and revert to increase before getting stable at 50nA after 20 days. It is interesting to reproduce the output current by the use of the electrochemical reaction parameters with inclusion of effects of weak interaction.

\section{RESULTS AND DISCUSSIONS}

The calculation of the output signal of the experiments was calculated by half - cell model. This model attempts to describe the time response evaluation of the output currents. the usual formulation of electrochemical reaction in a standar text book is adopted as a half-cell model, but the effect of the weak interaction is considered. Calculations were performed for the 
currents in Fig. 2. The most days, were treated as adjustable important parameter in the parameter for expressing initiation electrochemical model is the of beginning point of weakreaction rate coefficient $P_{c}$. interaction assistance. The ICP mass

Since there is the difference in measurement of ions in solution catalyst and operation temperature indicated that it took a few days for between the fuel cells and the ions such as $\mathrm{Ca}^{2+}, \mathrm{Mg}^{2+}$ to solve out present apparatus, the validity of $P_{c}$ into water. is not able to be discussed directly. The values of $t_{d}$ and $t_{r}$, namely few The values of $\mathrm{T}_{d}$ and $T_{r}$ are considered to include these

effects. The parameters $P_{c}, P_{a}, P_{i}, \quad{ }_{2} R_{\mathrm{HO}}$ simulations. 
and $k$

were fixed in all

Table 1

Parameters in the half-cell model analysis

\begin{tabular}{|c|c|c|}
\hline 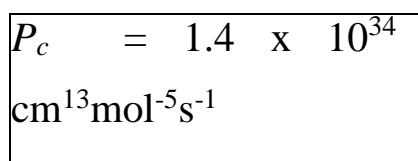 & $\begin{array}{l}R_{\mathrm{HO}}=2.24 \times 10^{-13} \\
\mathrm{~mol} \mathrm{~cm}{ }^{-3} \mathrm{~s}^{-1}\end{array}$ & $\begin{array}{l}k_{\mathrm{O}_{2}}= \\
0.12\end{array}$ \\
\hline $\begin{array}{l}P_{a}=1 \quad \mathrm{x} \quad 10^{-3} \\
\mathrm{~cm}^{4} \mathrm{~mol}^{-2} \mathrm{~s}^{-1}\end{array}$ & $\begin{array}{l}{\left[\mathrm{M}^{2+}\right]=2.04 \times 10^{-9}} \\
\mathrm{~mol} \mathrm{~cm}^{-3}\end{array}$ & $\begin{array}{l}T_{d}=2.00 \\
\mathrm{~d}\end{array}$ \\
\hline $\begin{array}{l}P_{i}=4.75 \\
10^{25} \mathrm{~cm}^{7} \mathrm{~mol}^{-3} \mathrm{~s}^{-1}\end{array}$ & $\begin{array}{l}{\left[\mathrm{O}_{2}\right]_{\mathrm{ini}}=1.09 \times 10^{-9}} \\
\mathrm{~mol} \mathrm{~cm}\end{array}$ & $\begin{array}{l}T_{r}=1.20 \\
\mathrm{~d}\end{array}$ \\
\hline
\end{tabular}

The parameters listed in Table $\mathbf{1}$ were applied for the analysis the concentration of ions and oxygen gas were taken to be adjustable in the calculation together with the time parameters $T_{d}$ and $T_{r}$. Current result of experimental and calculation data are plot in Fig. (2).

The initial peak around 1.5 days is considered to be ascribed to the reduction and oxydation of impurity ions, and the data after two days were considered in calculation. The experimental data are indicated by quadrangles, while solid line represents calculation one. The comparison suggest that the halfcell model is applicable to the experimental data.

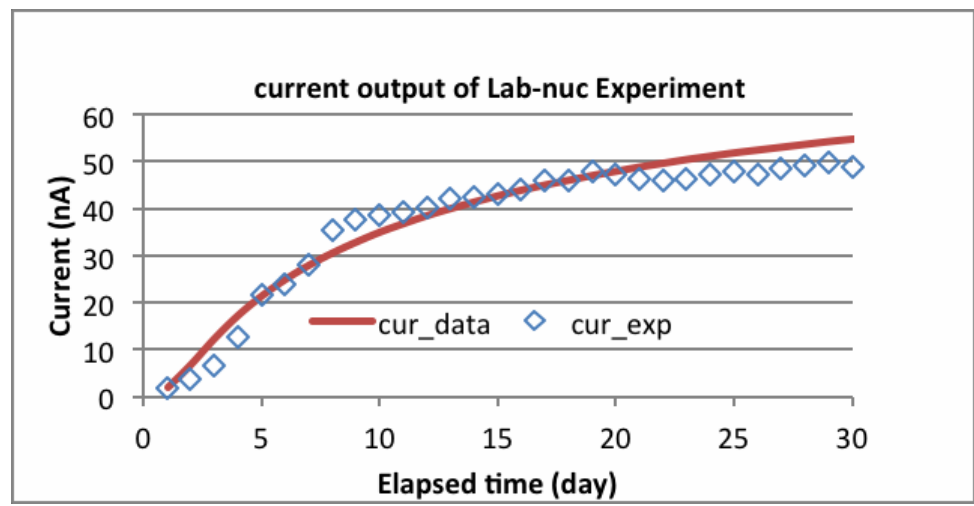


Fig. 2 Output current results versus time for experimental and theoretical data of Kyushu university laboratory.

We also calculate the corresponding solution for hydrogen ion and oxygen concentrations, as shown in Figs. 3. Previous study gives oxygen saturation about 8 per cent at the starting of experiment, and it was considered as a responds of impurity current. Hence, current of impurity is not described in the fitting. However, the results suggest that hydrogen ion is mostly saturated $1.4 \times 10^{-7}\left(\mathrm{~mol} \mathrm{~cm}^{-3}\right)$ after two weeks, instead of oxygen concentration remains $5 \times 10^{-8}(\mathrm{~mol}$ $\left.\mathrm{cm}^{-3}\right)$. the hydrogen ion concentration increases gradually because of dissociative ionization reaction between water and neutrino.

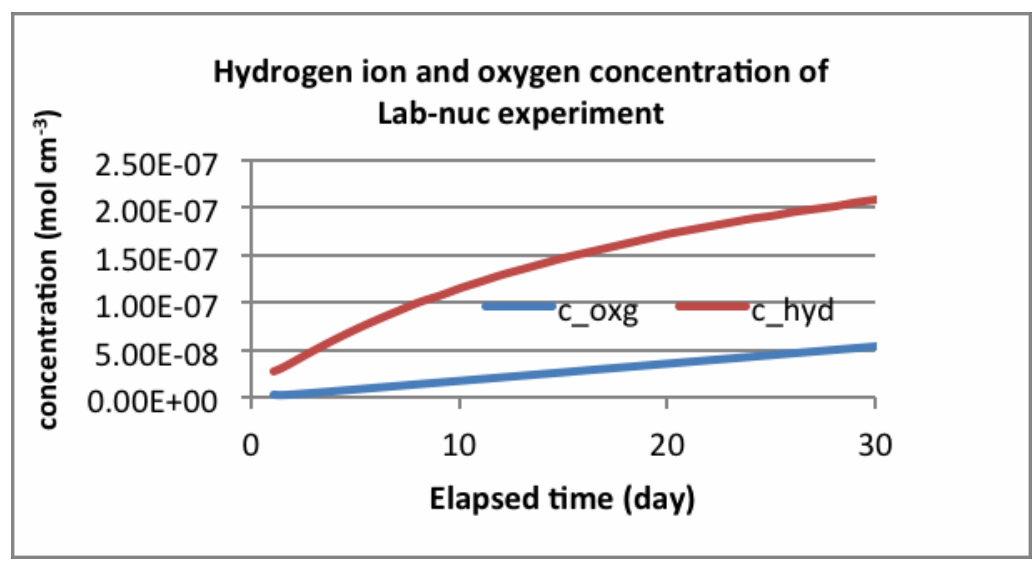

Fig. 3 Calculation result of hydrogen ion and oxygen concentrations of Kyushu university laboratory.

The reaction rate of water dissociative ionization is $R_{H O}=.5 \mathrm{x}$ $10^{-13} \mathrm{~mol} \mathrm{~cm}^{-3} \mathrm{~s}^{-1}$. This rate values implies that the interaction of neutrino and water molecules is similar to electromagnetic interaction at $\mathrm{keV}$ energy range. 
Therefore, the detector is sensitive presume for some keV energy range of neutrinos. The radioactive decay of natural elements (mainly ${ }^{238} \mathrm{U}$ and ${ }^{232} \mathrm{Th}$ ) in the interior of the KAMLAND

group, the geoneutrinos flux is about $f_{v}=5.7 \mathrm{x}$ $10^{-5}$

compare with the typical cross section of $\sigma \sim 10^{-43} \mathrm{~cm}^{2}(\mathrm{E} / \mathrm{MeV})$.

This increasing value perhaps comes from interaction of neutrino mass with skalar auxiliary field in raw silk. When neutrino breaks into boson and fermion pieces. This

It was found that correlation is given in

\section{CONCLUSION}

We attempted to study the process of neutrino interactions and water molecules in neutrino detector with the electrochemical and halfcell model with enclusion of weakinteraction effect. It was confirmed that, the output current are earth produces antineutrinos. It was believed that neutrinos are generated within earth's crust. According to the calculation by the

$$
\begin{aligned}
& \sigma \approx 10^{-19} \mathrm{~cm}^{-3} . \\
& \text { This value is very } \\
& \text { large when we }
\end{aligned}
$$

constituents particles become reactive particles. However, this analysis primarily ensures the influences of current response to water dissociative ionization rate, $R_{H o}$, especially in the reduction reaction of carbon electrode, $P_{c}$.

the form of $I \propto R_{H O} \ln P_{c}$.

generated by electrochemical reaction. We employ the model not only to solve output current of environmental neutrino, but also hydrogen ion concentrations. All calculations provide a compatible solution with experimental data.

\section{Acknowledgement}


The authors thank the Mr. Liu

Wei for providing any experimental data

\section{REFFERENCES}

C.L. Cowan Jr., Reines, et al. 1956.

Science 124.

http://www.awa.tohoku.ac.jp/ sans

hiro/research/geoneutrino/sp

ectrum/index.html.

Kenji Ishibashi, et al. 2011. Constituent motion of neutrino by internal weak interaction potential beyond the influence of Fermi constant.

Kenji Ishibashi, et al. 2012. Electic current induced by anti electron neutrinos in an electrochemical apparatus.

Liu Wei. 2004. Study on Detection of Environmental Neutrinos by Means of Miniaturized Apparatus Based on Electrochemical Reaction 54.

M.A. Halim. 2006. Experimental Study on Signal Behaviour of Electrochemical Detector for Environmental Neutrinos 74.

W.Pauli Jr. 1930. Address to group on radioactivity. 
